\title{
FIGO Stage IC
}

National Cancer Institute

\section{Source}

National Cancer Institute. FIGO Stage IC. NCI Thesaurus. Code C96251.

A FIGO stage term that applies to gynecologic cancers. For cervical cancer, there is no FIGO stage IC; for endometrial cancer, in the older FIGO classification stage scheme, it referred to invasion of more than a half of the myometrium. Stage IC for endometrial cancer has been eliminated in the most recent (2010) FIGO classification stage scheme. 